\title{
GEOMETRIC INEQUALITIES INVOLVING THE STRETCH OF A SET
}

\author{
by J. N. LILLINGTON*
}

(Received 21st July 1975)

\section{Introduction}

A number of papers have been written concerning the properties of triangles which circumscribe convex sets, see for example (1), (2), (3).

In this note we shall characterise two set functionals, one of which has already been introduced in (6). This will enable us to produce some new results in the spirit of those obtained in the papers mentioned above. These two functionals are interesting in that although they are defined in a rather abstruse manner, they turn out to have an intuitive geometric meaning, namely that they are the minimal widths of certain circumscribing triangles.

\section{Notation and definitions}

Unless otherwise stated, all sets $X$ are assumed to be compact convex subsets of the Euclidean plane $R$. If $x, y, z \in R$ we define points $w$ and $t$ as follows: suppose

1) $x, y, z$ are all distinct.

(a) If $x, y, z$ are the vertices of a triangle each of whose angles is less than $\frac{2}{3} \pi$ let $t=w=p$ be the unique point $p$ satisfying $\widehat{x p y}=\widehat{x p z}=$ $\widehat{y p z}=\frac{2}{3} \pi$.

(b) If $\widehat{y} \hat{x z}$ say, is equal to $\frac{2}{3} \pi$ let $w=t=x$.

(c) If $\widehat{y x z}$ say, is greater than $\frac{2}{3} \pi$ let $w=x$ and let $t$ be the unique point $\dagger$ lying on the same side of $y z$ as $x$ such that $\widehat{y t x}=\widehat{z t x}=\frac{1}{3} \pi$.

Otherwise

2) $x, y, z$ are not all distinct and $x=y$ say.

Then let $w=t=x$.

For any two points $x^{\prime}, y^{\prime} \in R$, let $x^{\prime} y^{\prime}$ denote the segment joining $x^{\prime}$ and $y^{\prime}$ and also the distance between $x^{\prime}$ and $y^{\prime}$. Let $\phi(x, y, z)$ and $\psi(x, y, z)$

\footnotetext{
* This work was carried out while the author was a research student at Royal Holloway College, London and is a revised version of part of the author's thesis approved for the Ph.D. degree.

t If $\widehat{y x z}=\pi$, then strictly speaking $t$ is not distinct and can be one of two points which are reflections of each other in $y z$. However, $K(x, y, z)$ is still well-defined.
} 
denote the set union of the segments $w x, w y, w z$ and $t x, t y, t z$ respectively. We shall call $w$ the centre of connexion of $\phi(x, y, z)$ and $t$ the centre of revolution of $\psi(x, y, z)$. Further write

$$
\begin{aligned}
L(x, y, z) & =w x+w y+w z \\
\text { and } K(x, y, z) & =t x+t y+t z \text { if case } 1(\mathrm{a}) \text { is applicable, } \\
& =-t x+t y+t z \text { otherwise. }
\end{aligned}
$$

The reader will note that $L(x, y, z)$ is the connected set of minimal length (linear measure) containing $x, y, z$ as defined in (6) and that $w$ and $\phi(x, y, z)$ are also as defined in that paper. It is also convenient to write $x^{\prime} y^{\prime} z^{\prime}$ to denote the convex hull of any three points $x^{\prime}, y^{\prime}, z^{\prime}$.

The stretch of $x$ with respect to $X$ is defined to be

$$
L(X ; x)=\sup _{y, z \in X} L(x, y, z)
$$

and the stretch of $X$ is defined to be

$$
L(X)=\sup _{x \in X} L(X ; x)
$$

Similarly, the equilateral stretch $K(X ; x)$ of $x$ with respect $X$ is defined by using $K(x, y, z)$ and the equilateral stretch $K(X)$ of $X$ follows.

If $\mathscr{K}$ is the class of compact sets in the plane we note that it follows from definition that the functions $L$ and $K$ are continuous over $\mathscr{K}$ under the Hausdorff metric.

\section{Preliminary lemmas}

Lemma 1. If $x, y, z \in \mathbb{R}$, let $t$ be the centre of revolution of $\psi(x, y, z)$. Let $u$ be the third vertex of the equilateral triangle of uyz which lies on the side of $y z$ opposite to $x$ with the convention that if $y=z$, then $u=y=z$. Then

1) The line segments tx and ux lie on a line,

2) $K(x, y, z)=u x$.

Proof. By standard continuity arguments it is sufficient to prove the lemma, with the assumption that $x, y, z$ are the vertices of a triangle. The case where $x, y, z$ form the vertices of a triangle, each of whose angles is less than $\frac{2}{3} \pi$ has already been proved in (4) on page 63 . The proof of the remaining case involves similar elementary geometric arguments and is omitted.

Corollary. Let $x, y, z$ be distinct points in $R$. Then, in the notation of Lemma 1, 


$$
\begin{aligned}
& K(x, y, z)>y z \text { if } \widehat{y x z}<\frac{5}{6} \pi \text {, } \\
& =y z \text { if } \widehat{y x z}=\frac{5}{6} \pi \text {, } \\
& <y z \text { if } \widehat{y x z}>\frac{5}{6} \pi \text {. }
\end{aligned}
$$

Proof. Let $\lambda=y z$. We note that $u x=y z=\lambda$ if and only if $x$ lies on the circle centre $u$ and radius $\lambda$. The corollary is then obvious.

Lemma 2. Let $x, y, z$ be the vertices of a triangle $T$. Then the equilateral triangle of maximal height which circumscribes $T$ and whose boundary contains the vertices of $T$ is unique and has height $K(x, y, z)$.

Proof. Suppose $S$ is an equilateral triangle which circumscribes $T$ and whose boundary contains the vertices of $T$, of side-length $s$ and area $\Delta$. Let $\psi(x, y, z)$ have centre of revolution $t$. We may assume the segments $t x, t y$, $t z$ make an acute angle $\theta$ with the sides of $S$ containing $x, y, z$ respectively, whenever of course each segment is defined. Then if each angle of $T$ is less than $\frac{2}{3} \pi$,

$$
2 \Delta=s(t x+t y+t z) \sin \theta .
$$

Otherwise we may assume that the angle $\widehat{y x z}$ say is greater than or equal to $\frac{2}{3} \pi$. In this case

$$
2 \Delta=s(-t x+t y+t z) \sin \theta
$$

Thus by definition

$$
2 \Delta=s \cdot K(x, y, z) \sin \theta,
$$

and the lemma is proved on noting that $S$ has maximal height if and only if $\theta=\frac{1}{2} \pi$.

Lemma 3. In the notation of Lemma 2, the triangle of maximal minimal width which circumscribes $T$ and whose boundary contains the vertices of $T$, is unique, isosceles with its two equal sides at least as long as the third side, and has minimal width $L(x, y, z)$.

Proof. Let $w$ be the centre of connexion of $\phi(x, y, z)$. We define a triangle $Q$ as follows:

If each angle of $T$ is less than $\frac{2}{3} \pi$, let $Q$ be the equilateral triangle bounded by the lines through $x, y, z$ perpendicular to $w x, w y, w z$ respectively.

If the angle $\widehat{y x z}$ say of $T$ is greater than or equal to $\frac{2}{3} \pi$ let $Q$ be the isosceles triangle bounded by the lines through $y, z$ perpendicular to $w y, w z$ respectively and the external bisector of $\widehat{y x z}$.

Then in both cases $Q$ is isosceles with its two equal sides at least as 
long as the third side and has minimal width $L(x, y, z)$. Next, let $S^{\prime}$ be a triangle of maximal minimal width $\lambda$ which circumscribes $T$ and whose boundary contains the vertices of $T$. We note that $S^{\prime}$ is not an infinite strip of width $\lambda$ for if this were the case, then $\lambda$ would not exceed the diameter of $T$, which in turn is less than $L(x, y, z)$. But by definition $\lambda \geqslant L(x, y, z)$. Thus $S^{\prime}$ is a proper triangle. Further for the same reason, each vertex of $T$ is contained in at most one side of $S^{\prime}$. Thus if $S^{\prime}$ is not isosceles with its two equal sides at least as long as the third side then we may assume that $S^{\prime}$ has sides of length $s_{1}, s_{2}, s_{3}$ containing $x, y, z$ in their relative interiors respectively where

$$
s_{3}>s_{2} \geqslant s_{1} \text {. }
$$

But then we may obtain a new triangle of minimal width greater than $\lambda$, by applying a suitably small rotation to the side of length $s_{1}$ about $x$. This however contradicts the definition of $S^{\prime}$ and so $S^{\prime}$ has the stated property.

We may assume then that

$$
s_{3}=s_{2} \geqslant s_{1} \text {. }
$$

Thus if the area of $S^{\prime}$ is $\Delta^{\prime}$, then

$$
\begin{aligned}
2 \Delta^{\prime}=s_{2} \lambda & \leqslant s_{1} \cdot w x+s_{2} \cdot w y+s_{3} \cdot w x \\
& \leqslant s_{2}(w x+w y+w z) \\
& =s_{2} \cdot L(x, y, z)
\end{aligned}
$$

Hence it follows that

$$
\lambda \leqslant L(x, y, z)
$$

with equality if and only if $S^{\prime}$ is the triangle $Q$ as defined earlier. The lemma is proved.

Note. We note that if $x, y, z$ are not all distinct and say $x=y \neq z$ then the equilateral triangle of maximum height which circumscribes $y z$ has height $y z=K(x, y, z)$ and the triangle of maximal minimal width with this property also has minimal width $y z=L(x, y, z)$. Thus Lemmas 2 and 3 remain true in this sense.

Remarks. Before stating the next lemma it is convenient to note the following:

1) If $L(X)=L(x, y, z)$ for $x, y, z \in X$, then $x, y, z$ are necessarily contained in $\partial X$ (the frontier of $X$ ). Further $x, y, z$ are collinear if and only if $X$ is a segment. This is an immediate consequence of the definition.

2) If $K(X)=K(x, y, z)$ for $x, y, z \in X$, then again $x, y, z \in \partial X$. Also either $x, y, z$ form the vertices of a triangle, each of whose angles is less than $\frac{5}{6} \pi$ or the points $x, y, z$ are not all distinct. To see this suppose that 
$\widehat{y x z}>\frac{5}{6} \pi$ where the points are assumed to be distinct. It then follows from the Corollary to Lemma 1 that

$$
K(X)=K(x, y, z)<y z=K(y, y, z) \leqslant K(X),
$$

which is impossible. The statement follows. Following (6), we shall say that a support line $l$ to $X$ supports $X$ regularly or that $l$ is a regular support line to $X$ if $l$ meets $\partial X$ in a single point.

Lemma 4. Suppose $K(X)=K(x, y, z)$ for some $x, y, z \in \partial X$. Then 1) If $x, y, z$ are the vertices of a triangle, let $\psi(x, y, z)$ have centre of revolution $t$ and let $v$ be any vertex of triangle $x y z$. Then if $t \neq v$ the line through $v$ perpendicular to tv supports $X$ regularly and if $t=v$ then the external bisector of the obtuse angle between the two lines of the triangle incident at $v$ supports $X$ regularly.

2) If $x, y, z$ are not all distinct we may assume $x=y \neq z$. Then all lines through $y$ or $z$ which make an acute angle of $\frac{1}{6} \pi$ with yz support $X$ regularly.

Proof. 1) Suppose, without loss in generality, that $v=x$. On $y z$ erect the equilateral triangle $u y z$ with $u$ on the side of $y z$ opposite to $x$. Let $l$ be the line through $x$ perpendicular to $t x$ if $t \neq x$ and the external bisector of $\widehat{y x z}$ if $t=x$. By Lemma $1, l$ is perpendicular to $u x$. Now if $l$ does not support $X$ regularly then there exists $x^{\prime} \in X \cap l$ on the side of $y z$ opposite to $u$ and $x^{\prime} \neq x$. But then again by Lemma 1 ,

$$
K(X) \geqslant K\left(x^{\prime}, y, z\right)=u x^{\prime}>u x=K(x, y, z)=K(X)
$$

which is contradictory. Thus the first part is proved.

2) Suppose $x=y \neq z$. It is sufficient only to consider the point $y$. Let $l$ be the line through $y$ making an acute angle of $\frac{1}{6} \pi$ with $y z$, and suppose $l$ does not support $X$ regularly. Then there exists a point $y^{\prime}$ on $X \cap l$ and $y^{\prime} \neq y$. By erecting the equilateral triangle $u^{\prime} y y^{\prime}$ with $u^{\prime}$ on the side of $y y^{\prime}$ opposite to $z$ and applying Lemma 1 , it is easy to see that

$$
K(X) \geqslant K\left(y^{\prime}, y, z\right)=u^{\prime} z>y z=K(x, y, z)=K(X),
$$

again contradicting the definition of $K(X)$. Thus $l$ is a regular support line and the proof of the lemma is complete.

Lemma 5. If for any set $X$ with a non-empty interior, $L(X)=L(x, y, z)$, where $x \in \partial X$, if $\phi(x, y, z)$ has centre of connexion $w$, and if $v$ is any vertex of triangle $x, y, z$ then if $w \neq v$ the line through $v$ perpendicular to wv supports $X$ regularly and if $w=v$ then the external bisector of the obtuse angle between the two lines of the triangle incident at $w$ supports $X$ regularly.

Proof. This lemma was proved in detail in (6) and the proof is omitted. 


\section{Geometric interpretation}

We are now in a position to give the geometric interpretation of the stretch and equilateral stretch of $X$.

Theorem 1. 1) The equilateral triangle of maximal height circumscribing $X$ has height $K(X)$.

2) The triangle of maximal minimal width circumscribing $X$ has minimal width $L(X)$.

Proof. If $X$ is a segment then the theorem is true by the note after Lemmas 2 and 3. Thus suppose that $X$ has a non-empty interior.

We shall prove parts 1) and 2) simultaneously. Suppose that the equilateral triangle $S$ of maximal height circumscribing $X$ has height $\lambda$ and that the triangle $S^{\prime}$ of maximal minimal width circumscribing $X$ has minimal width $\mu$. We may assume the points $x, y, z \in \partial X$ lie one on each side of $S$ and the points $x^{\prime}, y^{\prime}, z^{\prime} \in \partial X$ lie one on each side of $S^{\prime}$. By Lemmas 2 and 3 respectively

and

$$
\lambda \leqslant K(x, y, z)
$$

$$
\mu \leqslant L(x, y, z) .
$$

Otherwise suppose $K(X)=K(x, y, z)$ for some $x, y, z \in \partial X$ and $L(X)=$ $L\left(x^{\prime}, y^{\prime}, z^{\prime}\right)$ for some $x^{\prime}, y^{\prime}, z^{\prime} \in \partial X$. Then by Lemmas 4 and 5 respectively, there exists an equilateral triangle of height $K(x, y, z)$ circumscribing $X$ and a triangle of minimal width $L(x, y, z)$ circumscribing $X$. Thus

and

$$
\lambda \geqslant K(x, y, z)
$$

$$
\mu \geqslant L(x, y, z) \text {. }
$$

Equations (1), (2), (3) and (4) imply the result. We also note that $S^{\prime}$ has the property stated in Lemma 3.

Before proving our next theorem we need another lemma.

Lemma 6. Let $X$ be a compact set which is not necessarily convex and suppose $Y$ is the convex hull of $X$. Then

$$
\text { 1) } \quad K(X)=K(Y)
$$

Proof. If $Y$ is a segment the lemma is trivial and we suppose that $Y$ has a non-empty interior. If 1 ) is false then $K(Y)>K(X)$. Thus we may suppose $K(x, y, z)=K(Y)$ where $x \in Y$ and $x \notin X$. By Lemma 4, there exists a regular support line $l$ to $Y$ at $x$. Thus $l \cap X=\emptyset$. But this implies that $l \cap Y=\emptyset$ which is contradictory. Thus $K(Y)=K(X)$ and similarly $L(Y)=L(X)$. 
Corollary. Let $x, y, z \in R$.

1) (a) If $x, y, z$ form the vertices of a triangle each of whose angles is less than $\frac{5}{6} \pi$,

$$
K(x y z)=K(x, y, z)>\max (x y, x z, y z) .
$$

(b) If $\widehat{y x z}$ say, is equal to $\frac{5}{6} \pi$ or $x, y, z$ are not distinct and $x=y$ then

$$
K(x y z)=K(x, y, z)=y z>\max (x y, x z) .
$$

(c) If $\widehat{y x z}$ say is greater than $\frac{5}{6} \pi$, then

$$
K(x y z)=y z>\max (x y, x z, K(x, y, z))
$$

2) $L(x y z)=L(x, y, z) \geqslant \max (x y, x z, y z)$

with equality if and only if $x y z$ is a segment.

Proof. The proof follows for 1) by Lemma 6 and the Corollary to Lemma 1. The proof for 2) is immediate from definition.

In view of Theorem 1, it is natural to ask the following question: For any set $X$, what is the upper bound for the minimal widths of triangles circumscribing $X$ relative to the height of the maximal circumscribing equilateral triangle? We prove

Theorem 2. Let $\lambda$ be the height of the maximal equilateral triangle circumscribing $X$. Then every circumscribing triangle has minimal width less than or equal to $2 \lambda(2+\sqrt{ } 3)^{-1 / 2}$. fying

Proof. By the Blaschke selection theorem, there exists a set $Y$ satis-

$$
\frac{L(Y)}{K(Y)}=\sup _{X \in \mathscr{T}} \frac{L(X)}{K(X)} .
$$

Let $T$ be the isosceles triangle $x y z$ where $\widehat{y x z}=\frac{5}{6} \pi$ and $x y=x z$. By the Corollary to Lemma $6, L(T)=x y+x z$ and $K(T)=y z$. Then calculation gives

$$
\frac{L(Y)}{K(Y)} \geqslant \frac{L(T)}{K(T)}=\sec \frac{\pi}{12}=\frac{2}{(2+\sqrt{ } 3)^{1 / 2}} .
$$

We may choose points $x, y, z \in Y$ such that

$$
L(x y z)=L(x, y, z)=L(Y) .
$$

Since also $K(x y z) \leqslant K(Y)$ it follows from (5) that we may assume $Y=x y z$. Now $Y$ is not a segment and not a triangle each of whose angles is less than or equal to $\frac{2}{3} \pi$ for if this were so then by the definition of $L(x, y, z)$ and $K(x, y, z)$ and the Corollary to Lemma 6 ,

$$
\frac{L(Y)}{K(Y)}=\frac{L(x, y, z)}{K(x, y, z)}=1
$$


which contradicts (6). Thus we may suppose that $Y$ is a triangle and $\widehat{y x z}>\frac{2}{3} \pi$. We shall prove next that $x y=x z$.

On $y z$ erect the equilateral triangle $u y z$ with $u$ on the side of $y z$ opposite to $x$. Suppose that the line $l$ through $x$ perpendicular to $u x$ makes acute angles $\alpha, \beta$ with $x y, x z$ respectively. Let $x^{\prime}$ be a point on $l$ distant $\delta$ from $x$. (It is convenient to take $\delta>0$ if $x^{\prime}$ lies on the same side of $u x$ as $y$ and $\delta<0$ otherwise). Calculation gives

$$
\begin{aligned}
& u x^{\prime}=u x+O\left(\delta^{2}\right) \\
& x^{\prime} y=x y-\delta \cos \alpha+O\left(\delta^{2}\right) \\
& x^{\prime} z=x z+\delta \cos \beta+O\left(\delta^{2}\right) .
\end{aligned}
$$

Then using the Corollary to Lemma 6

$$
\begin{aligned}
\frac{L\left(x^{\prime} y z\right)}{K\left(x^{\prime} y z\right)} & =\frac{x^{\prime} y+x^{\prime} z}{\max \left(u x^{\prime}, y z\right)} \\
& =\frac{x y+x z}{\max (u x, y z)}+\frac{\delta(\cos \beta-\cos \alpha)}{\max (u x, y z)}+O\left(\delta^{2}\right) . \\
& =\frac{L(Y)}{K(Y)}+\frac{\delta(\cos \beta-\cos \alpha)}{\max (u x, y z)}+O\left(\delta^{2}\right) .
\end{aligned}
$$

Hence by a suitable choice of $\delta$ we may contradict the definition of $Y$ in (5) unless $\alpha=\beta$. Since $\widehat{y x z}>\frac{2}{3} \pi$ it follows that $x y=x z$. Now if $\widehat{y x z}>\frac{5}{6} \pi$, then we may choose a point $x^{\prime \prime}$ such that $y x^{\prime \prime} z>\frac{5}{6} \pi$ and the perimeter of $x^{\prime \prime} y z$ exceeds the perimeter of $y x z$. Then

$$
\begin{aligned}
\frac{L\left(x^{\prime \prime} y z\right)}{K\left(x^{\prime \prime} y z\right)} & =\frac{x^{\prime \prime} y+x^{\prime \prime} z}{y z}>\frac{x y+x z}{y z} \\
& =\frac{L(Y)}{K(Y)}
\end{aligned}
$$

which again contradicts (5). Thus $\frac{2}{3} \pi<\widehat{y x z} \leqslant \frac{5}{6} \pi$. Now if $\widehat{y x z}<\frac{5}{6} \pi$, let $l^{\prime}$ be the external bisector of $\widehat{y x z}$, and let $m^{\prime}, n^{\prime}$ be the lines through $y, z$ perpendicular to $t y, t z$ respectively. Choose points $y^{\prime}, z^{\prime}$ on $m^{\prime}, n^{\prime}$ respectively satisfying $\widehat{y x z}<\widehat{y^{\prime} x z^{\prime}}<\frac{5}{6} \pi$ and $y y^{\prime}=z z^{\prime}$. Then $K(x, y, z)=$ $K\left(x, y^{\prime}, z^{\prime}\right)$. Hence this implies

$$
\begin{aligned}
\frac{L\left(x y^{\prime} z^{\prime}\right)}{K\left(x y^{\prime} z^{\prime}\right)} & =\frac{x y^{\prime}+x z^{\prime}}{K\left(x, y^{\prime}, z^{\prime}\right)}>\frac{x y+x z}{K(x, y, z)} \\
& =\frac{L(Y)}{K(Y)}
\end{aligned}
$$


which is again impossible. Thus we have shown that if $Y$ is a triangle then $Y$ is a triangle $x y z$ where $\widehat{y x z}=\frac{5}{6} \pi$ and $x y=x z$. Hence equality holds in (6) and the theorem is proved.

It is easy to see that although the result is best possible, there are many extremal figures $Y$. We remark that we can also use a similar method to give the upper bound for the minimal widths of triangles circumscribing a set $X$ in terms of the perimeter of $X$, see Theorem 1 in (2).

Following the definition of a completely stretched set in (6), we shall say that a set $X$ of equilateral stretch $\lambda$ is completely equilaterally stretched if $x \notin X$ implies $K(X ; x)>\lambda$.

Theorem 3. The following statements are equivalent.

(a) $K(X ; x)=\lambda$ for each $x \in \partial X$,

(b) $X$ is completely equilaterally stretched with stretch $\lambda$,

(c) $X$ is a rotor for an equilateral triangle of height $\lambda$.

Proof. In Theorem 3 in (6) it was shown that if $X$ could be circumscribed by an equilateral triangle $S$ of height $\lambda$, one of whose sides supported $X$ at $x$ for each $x \in \partial X$, then $X$ was necessarily a rotor for $S$. Thus by Lemma 4 it follows that (a) implies (c).

To show (c) implies (b) suppose $x \notin X$. Let $x^{\prime}$ be the point of $\partial X$ nearest $x$. Since $X$ is a rotor for an equilateral triangle $S$, there is an orientation of $S$ such that one side $s_{1}$ of $S$ supports $X$ at $x^{\prime}$ and is perpendicular to $x x^{\prime}$. Suppose that $y^{\prime}, z^{\prime}$ are the points of contact of the other two sides $s_{2}, s_{3}$ of $S$ respectively in this orientation. If follows by Lemma 2 that

$$
K(X)=K\left(x^{\prime}, y^{\prime}, z^{\prime}\right)=\lambda .
$$

Further the equilateral triangle $S^{\prime}$ bounded by the line through $x$ parallel to $s_{1}$, and the lines $s_{2}, s_{3}$ clearly circumscribes $x y^{\prime} z^{\prime}$ and has height $\mu>\lambda$.

Hence by the definition of $K(X ; x)$ and Lemma 2 it follows that

$$
K(X ; x) \geqslant K\left(x, y^{\prime}, z^{\prime}\right) \geqslant \mu>\lambda=K(X) .
$$

Finally to show (b) implies (a) we can apply the same continuity argument as was used in Theorem 1 in (6) to show that $X$ completely stretched with stretch $\lambda$ implies that $L(X ; x)=\lambda$ for each $x \in \partial X$. Thus the proof of Theorem 3 is complete.

In (7) Fujiwara and Kakeya gave an analytic characterisation of the rotors for an equilateral triangle. Theorem 3 is interesting in that the characterisation depends on purely geometric notions. The reader will also note that Theorem 3 is an analogue of Theorem 1 in (6) and in our next theorem we state a result directly related to Theorem 2 in that paper. 
Theorem 4. If $S$ is the maximal equilateral triangle circumscribing $X$ then there exists a set $Y$ which contains $X$ and which is a rotor for $S$.

Proof. The proof that a set $X$ of equilateral stretch $\lambda$ is contained in a completely equilaterally stretched set $Y$ of equilateral stretch $\lambda$ is identical to the proof of Theorem 2 in (6). Theorem 4 then follows from Theorem 3.

For the remainder of this paper we shall write $P(X), H(X)$ and $A(X)$ to denote the perimeter, minimal width and area of $X$ respectively.

Corollary. If $X$ has perimeter $\lambda$, minimal width $\delta$ or area $\sigma$ then the maximal equilateral triangle circumscribing $X$ has height greater than or equal to $3 \lambda / 2 \pi, 3 \delta / 2$ or $3 \sigma^{1 / 2} / \pi^{1 / 2}$, respectively, with equality if and only if $X$ is a rotor for an equilateral triangle, a rotor for a regular hexagon, or a disc.

Proof. We have to show 1) $K(X) / P(X) \geqslant 3 \lambda /(2 \pi)$, 2) $K(X) / H(X) \geqslant$ $3 / 2$ and 3) $(K(X))^{2} / A(X) \geqslant 9 / \pi$ with equalities as stated in the theorem.

To prove 1) we note that if $K(X) / P(X)$ is minimal then, by Theorem 4, $X$ is necessarily a rotor for an equilateral triangle and so the result follows by (7) page 106. Result 2) follows from 1) and the well known result $P(X) / H(X) \geqslant \pi$ with equality if and only if $X$ is a set of constant width. Finally result 3) follows from 1) and the well known isoperimetric inequality $P(X)^{2} / A(X) \geqslant 4 \pi$ with equality if and only if $X$ is a disc.

\section{Minimal Widths}

We finish by giving two results concerning the minimal widths of triangles circumscribing sets of constant width and sets which are rotors for an equilateral triangle.

Theorem 5. Let $X$ be a set of constant width $\lambda$. Then every triangle circumscribing $X$ has minimal width at most $\lambda \sqrt{ } 3$ with equality only possible if $X$ is a Reuleaux triangle.

Proof. We shall show first that the triangle $T$ of maximal minimal width circumscribing $X$ is necessarily equilateral.

If this is not so then we may assume there exist points $x, y, z$ which form the vertices of a triangle with $\widehat{y x z}>\frac{2}{3} \pi$, where $L(X)=L(x, y, z)=$ $x y+x z$. (Otherwise $K(X)=L(X)$.) Then it is clear, since $X$ has diameter $\lambda$ that $x y+x z$ is smaller than or equal to the sum of the two equal sides of an isosceles triangle with base $\lambda$ and base angles of $\frac{1}{6} \pi$. Hence

$$
x y+x z \leqslant \frac{2 \lambda}{\sqrt{ } 3} .
$$

But by (5) page 125, the radius of the incircle $I$ of $X$ is at least $(1-1 / \sqrt{ } 3) \lambda$.

Then if $o$ is the centre of this circle we may choose points $x^{\prime}, y^{\prime}, z^{\prime}$ on $I$ 
satisfying $\widehat{x O y^{\prime}}=\widehat{x^{\prime O z}} z^{\prime}=\widehat{y^{\prime O z} z^{\prime}}=\frac{2}{3} \pi$. Then by definition

$$
\begin{aligned}
L(X) & \geqslant L\left(x^{\prime}, y^{\prime}, z^{\prime}\right) \\
& \geqslant 3(1-1 / \sqrt{ } 3) \lambda \\
& >2 \lambda / \sqrt{ } 3 \\
& \geqslant x y+x z \text { by }(7) \\
& =L(X)
\end{aligned}
$$

which is contradictory. We suppose then that the points $x, y, z$ of $\partial X$ lie one on each side of the equilateral triangle $T$ of height $L(X)$. Suppose $x^{\prime \prime}$, $y^{\prime \prime}, z^{\prime \prime}$ are the midpoints of the sides containing $x, y, z$ respectively. Then $\max (x y, x z, y z)$ is minimal, if $x, y, z$ are allowed to vary on their respective sides, if and only if $x=x^{\prime \prime}, y=y^{\prime \prime}$ and $z=z^{\prime \prime}$. Thus by calculation,

$$
\frac{L(X)}{\lambda}=\frac{L(X)}{H(X)} \leqslant \frac{L(X)}{\max (x y, x z, y z)} \leqslant \sqrt{ } 3,
$$

with equality if and only if $x=x^{\prime \prime}, y=y^{\prime \prime}, z=z^{\prime \prime}$ and

$$
H(X)=x^{\prime \prime} y^{\prime \prime}=x^{\prime \prime} z^{\prime \prime}=y^{\prime \prime} z^{\prime \prime}=\lambda \text {. }
$$

Finally if equality holds in (8), let $Y$ be the Reuleaux triangle of constant width $\lambda$ bounded by the small $\operatorname{arcs} x^{\prime \prime} y^{\prime \prime}, x^{\prime \prime} z^{\prime \prime}, y^{\prime \prime} z^{\prime \prime}$ of radius $\lambda$. Then by for example Lemma 1, page 82 in (8), it follows that $Y \subset X$ and hence that $X=Y$ since $X$ and $Y$ both have constant width $\lambda$. The theorem is proved.

We now define the thin circular rotor for an equilateral triangle of height $\lambda$ to be the set bounded by the closed convex curve consisting of two circular arcs of radii $\lambda$, and for which the distance between the two angular points is equal to $\lambda$.

Theorem 6. Let $X$ be a set with minimal width $\lambda$ which is a rotor for an equilateral triangle $S$. Then every triangle circumscribing $X$ has minimal width at most $2(2+\sqrt{ } 3)^{1 / 2} \lambda$ with equality only possible if $X$ is the thin circular rotor.

Proof. We prove first that if $S$ has height $\mu$, then

$$
\mu \leqslant(2+\sqrt{ } 3) \lambda
$$

with equality if and only if $x$ is the thin circular rotor. Let $x, x^{\prime}$ be points for which $\lambda=x x^{\prime}$. Let $Y$ be the set bounded by the closed convex curve consisting of two circular arcs of radius $\mu$ which contain $x$ and $x^{\prime}$ respectively and whose centres lie on the line containing $x x^{\prime}$. We shall show next that $Y$ contains $X$. Suppose this is not so and that $x^{\prime \prime} \in X, x^{\prime \prime} \notin Y$ and, without loss in generality, that $x^{\prime \prime}$ lies on the same side of the bisector of $x x^{\prime}$ as $x$. Then since $X$ is a rotor for an equilateral triangle of height $\mu$, there exist points $y, z \in X$ 
such that

$$
K(x, y, z)=K(X)=\mu
$$

and such that the line $u x$ contains $x x^{\prime}$ where $u$ is the vertex of the equilateral triangle $u y z$ on the side of $y z$ opposite to $x$. But then

$$
\begin{aligned}
K(X)=\mu=K(x, y, z) & =u x \\
& <u x^{\prime \prime} \\
& =K\left(x^{\prime \prime}, y, z\right) \\
& \leqslant K(X)
\end{aligned}
$$

which is contradictory. Thus $Y$ contains $X$. Now if $\mu>(2+\sqrt{ } 3) \lambda$ then calculation gives

$$
P(Y)<2 \pi \mu / 3
$$

which implies

$$
P(X)<2 \pi \mu / 3 \text {. }
$$

But this implies $X$ is not a rotor for $S$, against hypothesis. Hence $\mu \leqslant$ $(2+\sqrt{ } 3) \lambda$. Further if equality holds then $P(Y)=2 \pi \mu / 3$. Hence $P(X)=$ $P(Y)$ and $X=Y$. Thus statement (9) follows. We show finally that

$$
L(X) \leqslant 2 \mu /(2+\sqrt{ } 3)^{-1 / 2}
$$

with equality if and only if $X$ is the thin circular rotor. Theorem 6 then follows from statements (9) and (10).

We first note that no three points $x, y, z$ in the frontier of $X$ can lie on a circle of radius greater than $\mu$. For suppose this is the case and $y, x, z$ lie in order on an arc, which we may suppose subtends an angle less than $\frac{1}{3} \pi$ at the centre of the circle.

Suppose that $\mu=K(X)=K\left(x, y^{\prime}, z^{\prime}\right)$ say, and let $u^{\prime}$ be the vertex of the equilateral triangle $u^{\prime} y^{\prime} z^{\prime}$ on the side of $y^{\prime} z^{\prime}$ opposite to $x$. Then it is easy to see that

$$
\max \left(u^{\prime} y, u^{\prime} z\right)>u^{\prime} x=K(X) .
$$

In other words either $K\left(y, y^{\prime}, z^{\prime}\right)>K(X)$ or $K\left(z, y^{\prime}, z^{\prime}\right)>K(X)$. But this is impossible and so the statement is proved. The statement implies that if $Y$ is a circle of radius $\mu$ containing any two points in $\partial X$, then the small arc of $Y$ also lies in $X$. Now suppose $L(X)=L(x, y, z)$ for $x, y, z \in X$. Then by Theorem 2, it follows that

$$
\frac{L(X)}{\mu}=\frac{L(X)}{K(X)} \leqslant \frac{L(x y z)}{K(x y z)} \leqslant \frac{2}{(2+\sqrt{ } 3)^{1 / 2}}
$$

with equality if and only if $x y z$ is an isosceles triangle of diameter $\mu$ and with its two equal sides containing an angle of $\frac{5}{6} \pi$. Finally suppose that equality holds and $y z=\lambda$. By the statement proved in the previous 
paragraph the set $Z$ bounded by the small arc $y z$ of radii $\mu$ is contained in $X$ and is precisely the thin circular rotor of $S$. Hence $X=Z$ and the theorem is proved.

Note

In this paper we have consistently used the fact that rotors for an equilateral triangle all have the same perimeter. However this is not so for the completely stretched sets. It would therefore be interesting to have a proof of the following.

Conjecture. Let $X$ be a completely stretched set with stretch $\lambda$. Then the perimeter of $X$ is greater than $2 \lambda$ with equality if and only if $X$ is a segment. (If $X$ is a segment the perimeter of $X$ is defined to be twice its length.)

\section{REFERENCES}

(1) H. G. EgGLeston, Some properties of triangles as extremal convex curves, J. London Math. Soc. 28 (1953), 32-36.

(2) H. G. EGGLESTON, On triangles circumscribing plane convex sets, $J$. London Math. Soc. 28 (1953), 36-46.

(3) H. G. EgGLESTON, Sets of constant width contained in a set of given minimal width, Mathematika 2 (1955), 48-55.

(4) H. G. EGGLeston, On the projection of a plane set of finite linear measure, Acta Math. 99 (1958), 53-91.

(5) H. G. EgGleston, Convexity (Cambridge University Press, 1958).

(6) J. N. Lillington, Completely stretched sets, Proc. Cambridge Philos. Soc. 75 (1974), 37-43.

(7) M. FUJIWARA and S. KAKEYA, On some problems of maxima and minima for the curve of constant breadth and the in-revolvable curve of the equilateral triangle, Tôhuku Math. J. 11 (1917), 92-110.

(8) A. S. BESICOVITCH, Measure of asymmetry of convex curves (II): Curves of constant width, J. London Math. Soc. 23 (1948), 237-240.

73, NORTHPORT DRIVE

WAREHAM

DORSET 